\title{
The Value of Tumor Markers in Endometrial Carcinoma: Review of Literature
}

\author{
Cenk Yasa ${ }^{*}$, Ozguc Takmaz, Ozlem Dural, Suleyman Engin Akhan
}

Gynecology and Obstetric Department, Istanbul Medical School, Istanbul University, Istanbul, Turkey.

Email: *cenk_yasa@yahoo.com

Received April 18 ${ }^{\text {th }}$, 2013; revised May 20 ${ }^{\text {th }}, 2013$; accepted May 27 ${ }^{\text {th }}, 2013$

Copyright (C) 2013 Cenk Yasa et al. This is an open access article distributed under the Creative Commons Attribution License, which permits unrestricted use, distribution, and reproduction in any medium, provided the original work is properly cited.

\begin{abstract}
Endometrial cancer is the most common gynecologic malignancy in more developed countries. Most endometrial carcinomas cases are diagnosed at an early stage with a tumor confined to the uterine corpus. Although most patients are cured by surgery alone, about $15 \%$ - 20\% with no signs of locally advanced or metastatic disease at primary treatment recurs, with limited responsiveness to systemic therapy. Although it has a comparatively low mortality rate compared with other gynaecological cancers, it is capable of aggressive behaviour. Diagnosis at an early stage is the most important determinant of survival. Discovery of new diagnostic biomarkers/panels for early diagnosis of endometrial cancer is one of the main challenges of modern medicine. For endometrial cancers, there are no established serum markers. We will review the present knowledge regarding tumor markers, assessing how such markers could be applied to address in screening, diagnosis and monitoring of endometrial carcinoma.
\end{abstract}

Keywords: Endometrial Cancer; Tumor Markers

\section{Introduction}

Endometrial cancer is the most common gynecologic malignancy of women. And it is the 4th common cancer in women [1]. The incidence of endometrial cancer is arising whole over the world last 20 years [2]. Every year about 200,000 new endometrial cancer cases are diagnosed whole over the world and an estimated 50,000 women will die from this disease [3]. Endometrial cancer is diagnosed mostly in 6th or 7th decade and $70 \%$ of these cases are postmenopausal.

Although several different histologic subtypes of endometrial cancer are recognized, these are traditionally classified in two types, type 1 and type 2 [4]. Type 1 endometrial cancers comprise about $80 \%$ of all new cases of endometrial cancer and are histologically well or moderately differentiated, highly estrogen-dependent, and typically have favourable prognosis. Type 2 endometrial cancers are mostly low differentiated, include other subtypes, often with serous papillary or clear cell histology, estrogen independent and having worse prognosis. Although type 2 endometrial cancers make up only $10 \%-15 \%$ of all endometrial cancer cases, they are responsible for about $50 \%$ of all recurrences [5].

\footnotetext{
*Corresponding author.
}

Around 5\% - 10\% of endometrial carcinomas have a hereditary basis, with hereditary non-polyposis colorectal cancer which is the most common cause [6]. The risk factors relate to hormonal stimulation of the endometrium, such as unopposed estrogen treatment, polycystic ovarian disease and estrogen-producing tumors. Overweight is present in 50\% with endometrial carcinoma and the risk is linked to disturbances in hormone metabolism related to obesity [7]. Primiparity is a risk factor for endometrial cancer and therapy of breast cancer with tamoxifen have a 6 - 8 fold increased risk [8,9].

Tumor markers can be secreted by the tumor in excess of the normal tissue or cell phenotype. Sometimes, the molecule is uniquely specific to tumor phenotype or stem-cell phenotype. Tumor markers might be unique extracellular matrix or cell adhesion molecules, receptors, growth factors, cytokines or products of abnormal metabolism. Rarely, the marker molecules can be released by other tissues and organs in response to signals from tumor. Even the body's own antibodies against tumor markers can be markers. Tumor markers can be associated with patient diagnosis, prognosis, clinical management and follow-up. Ideally, a serum marker would only appear in the blood of patients with a true malignancy; the marker would correlate with tumor stage and re- 
sponse to treatment, and it could be easily, cheaply, and reproducibly measured. The serum marker would be used for the screening of healthy populations and of specific groups with higher risk factors. The marker would enable a diagnosis for a specific type of cancer, help determine prognostic factors and be used monitor the course of treatment, remission, and recurrence, while receiving surgery, radiation, chemical and immunological treatments [10].

In this review, we present an overview of the serum tumor markers in current use in endometrium cancer.

\section{CA 125}

Also known as Cancer Antigen 125 or Carbohydrate Antigen 125, it is a mucin glycoprotein that was identified using monoclonal antibody OC 125 , hence the name. CA 125 was originally identified by Bast et al. in the 1980s [11]. It is present in all humans and present in mesothelial cells of the pleura, pericardium, peritoneum and Mullerian epithelium derivatives such as tubal, endometrial, and endocervical cells. CA 125 is the most reliable serum marker for ovarian cancer. Elevation of serum CA 125 has been detected in a number of physiological and pathological conditions associated with endometrial proliferation, including menstrual cycle, pregnancy, endometriosis and endometrial carcinoma [12]. A single CA 125 determination provides no advance in the early detection of endometrial as well as ovarian carcinoma in asymptomatic postmenopausal women compared to transvaginal ultrasonography. The vast majority of women with an elevated CA 125 value have some reason other than an ovarian or endometrial malignancy for this finding [13].

In detail, raised CA 125 levels (>35 U/ml) have been reported in $11 \%$ - $33.9 \%$ of patients with endometrial cancer [14-16]. Ginath et al. found that $21.4 \%$ of 28 patients with endometrioid endometrial carcinoma had elevated serum CA 125, whereas the percentage of patients with positive tissue immunostaining for the antigen was 89.3\%, which appeared to suggest the presence of mechanisms preventing the access of CA 125 into the circulation [17].

Preoperative elevated serum CA 125 levels in endometrial cancer patients, correlated with stage of disease, lymph node metastases, depth of myometrial invasion, and positive peritoneal cytology [18].

However, one of the major factors influencing the clinical utility of serum CA 125 is the selection of appropriate reference cut-off values. In a study of with endometrial cancer patients, serum CA 125 levels, determined in a total of 112 patients, were elevated above $35 \mathrm{U} / \mathrm{ml}$ in 12 of 79 patients (15.2\%) with stage I, 4 of 12 patients (33.3\%) with stage II, 8 of 13 patients (61.5\%) with stage III, and all of 8 patients (100\%) with stage IV disease.
Good correlation between serum CA 125 levels and the clinical stage of the disease was found in this study [19]. Using the cut-off of $20 \mathrm{U} / \mathrm{ml}$, Kurihara et al. reported that serum CA 125 had a sensitivity of $69.0 \%$, specifity of $74.1 \%$, positive predictive value of $58.8 \%$ and negative predictive value of $81.6 \%$ for assessment of myometrial infiltration [20]. Takami et al. found that CA 125 levels in 291 normal postmenopausal women without hormone replacement therapy have a weak negative correlation with their age. Therefore, the normal range of CA 125 of postmenopausal women is lower than that of cycling women. According to the authors CA 125 levels, which apparently fall within the normal range but are high for the respective age, may suggest an extensive search for an endometrial malignancy [21].

Several studies investigated whether serum CA 125 assay may provide additional information in the preoperative assessment of endometrial carcinoma, and in particular in the identification of those patients with high risk of microscopic extrauterine spread who need a lymphadenectomy. Sood et al. have shown that the likelihood of extrauterine disease in patients with endometrial cancer would be less than 3\% when the preoperative CA 125 level is less than or equal to $20 \mathrm{IU} / \mathrm{ml}$ [22]. Yildiz et al. reported that with a cutoff value $20 \mathrm{U} / \mathrm{ml}$, the sensitivity and specifity of CA 125 assay for detecting advance stage of disease and lymh node metastases were $75 \%$ and $69.51 \%$, respectively [23].

The serum CA 125 level usually parallels the clinical course of disease [24]. In the study of Hakala et al., serum CA 125 reflects the clinical course of disease in 63\% of cases [15]. Cherchi et al. reported that, during the follow-up, CA 125 values > $35 \mathrm{U} / \mathrm{ml}$ were detected in 50\% of relapsed cases and only in $5.1 \%$ of disease-free cases [16].

However, the fact that serum CA 125 levels are often elevated in disease-free endometrial cancer patients who have undergone abdominal radiation should be kept in mind [25]. Mesothelial cells, an ovarian cell line that does not secrete CA 125, normal breast epithelium, and normal fibroblasts were exposed to 500 cGy of 6-MV photon irradiation. By measuring CA 125 concentrations in culture medium, the authors found that radiation stimulated CA 125 secretion in mesothelial cells up to 32 times over nonirradiated controls, whereas normal fibroblasts, breast epithelium, and the ovarian cancer cell line did not produce CA 125 in either the presence or absence of radiation. Thus, irradiated mesothelial cells are a potential source of serum CA 125 in patients who have received abdominal irradiation.

Elevated pretreatment serum CA 125 levels are often associated with a poor clinical outcome [26]. Scambia et al. found a statistically significant relationship between CA $125>65 \mathrm{U} / \mathrm{ml}$ and a shorter survival [14]. In a series 
of 99 patients, Lundstrom et al. observed that CA 125 levels $>35 \mathrm{U} / \mathrm{ml}$ correlated significantly with cancer death [27]. Conversely, Sood et al. reported that serum CA $125>35 \mathrm{U} / \mathrm{ml}$ was an independent predictor for poor survival [22].

The role of serum CA 125 in the management of patients with serous papillary carcinoma of the endometrium is controversial. According to Price et al. serum CA 125 assay may reflect advance stage disease and portend a poor prognosis, but have limited utility in monitoring the response to chemotherapy and may not predict recurrence in absence of other clinical signs. In their study including 15 patients undergoing platinum-based chemotherapy, all five patients who died of disease had clinical or radiographic evidence of tumor, which CA 125 elevation did not precede or predict, and moreover, one patient with advanced disease at presentation never had an elevated CA 125 level but died of disseminated disease 14 months after diagnosis [28]. Conversely, Abramovich et al. found elevated CA 125 levels before the start of chemotherapy in 13 of 16 patients with uterine serous papillary carcinoma. Of the 13 patients, eight (57\%) experienced either a major reduction or normalization of CA 125 levels following therapy, consistent with their clinical course at that point. As for the 11 patients who ultimately relapsed, eight (73\%) were found to have a rise in serum CA 125 which closely corresponded to, or proceeded, the clinical relapse. Therefore, according to the authors, CA 125 assay seems to be a useful marker of disease activity in patients with this malignancy [29].

\section{Other Tumor Markers}

The serum markers CA 19.9, CA 15.3, CA 72.4 and CEA levels are raised in endometrial cancer patients in $22 \%$ $24 \%, 24 \%-32 \%, 22 \%-32 \%$ and $14 \%-22 \%$ of the cases, respectively [14,16,30-33]. Scambia et al. found CA 15.3 levels > $30 \mathrm{U} / \mathrm{ml}$ in $47 \%$ of patients with occult stage III compared to $18 \%$ of those with surgical stages I and II disease. A significant relationship was detected between CA 15.3 positivity ( $>30$ and $>50 \mathrm{U} / \mathrm{ml}$ ) and shorter (14). Serial CA 15.3 levels showed a good correlation with the clinical behavior of disease. In the series of Cherchi et al., a statistically significant difference between intrauterine (96 cases) and extrauterine (16 cases) disease at surgical-pathological stage was noted for CA 15.3 (28.1\% vs $56.2 \%$ ), but not for CA 19.9 or CEA positivity. As for the concomitant determination of different markers, only the combination of CA 125 and CA 19.9 offered interesting results for post-treatment surveillance. In fact, the association of these markers had a high sensitivity (83.3\%) for the detection of recurrence, with only $12.8 \%$ of false positive cases [16].

Hareyama et al. found elevated CA 72.4 levels in
$31.9 \%$ of 72 patients with endometrial carcinoma. Multivariate analysis showed a significant correlation between serum antigen positivity and adnexal metastases. Seven patients had increased serum CA 72.4 and normal serum CA 125 and CA 19.9, and in four of them the disease had spread beyond the uterus [32].

YKL-40 is another potential tumour marker for endometrial cancer and was found to be elevated in $76 \%$ of patients. Preoperative serum YKL-40 levels may lead to the identification of high risk subsets of patients with worse clinical outcomes [34]. Further investigation of this promising endometrial cancer marker in larger studies is warranted.

HE4 mentioned in the context of ovarian cancer, has also been shown to be elevated in early stage endometrial cancer and is more sensitive than CA 125, though one may debate the need for an early marker as most cases are present at early stage due to symptoms, it may benefit patients with Lynch II syndrome or those on tamoxifen. One study showed it to be the single most acurrate marker, regardless of stage as compared to CA 125, CA 72.4, soluble mesothelin-related peptide or alone [35]. However, its role in predicting recurrence and response to therapy need to be determined.

Raised serum macrophage colony-stimulating factor (M-CSF) levels were found about three fourths of patients with endometrial carcinoma, a significantly higher percentage of pathologic values than observed for either CA 125 or aminoterminal propeptide of type III collagen and also with stage I or stage II disease as often as with stage III or IV disease. As a result M-CSF may be useful in the early diagnosis of endometrial carcinoma. Also elevated levels of M-CSF at presentation appear to be predictive of an agressive clinical course [36].

Beck et al. reported that the mean serum OVX1 levels measured with an enzyme-immunoassay were 2.00 $( \pm 1.32) \mathrm{U} / \mathrm{ml}$ in 192 patients with endometrial carcinoma compared to $1.34( \pm 0.74) \mathrm{U} / \mathrm{ml}$ in apparently healthy female. Applying a cut-off of $2.8 \mathrm{U} / \mathrm{ml}$, serum OVX1 was elevated in $19.7 \%$ of patients with stage I disease, $29.4 \%$ of those with stage II, $22.7 \%$ of those with stage III, and $36.4 \%$ of those with stage IV disease [19].

Significantly elevated levels of serum sFas were demonstrated in endometrioid adenocarcinoma of the endometrium compared to that of healthy women [37]. Dobrzycka and et al. found significant correlation between clinical stage, histological grade, 5-year disease-free survival and vascular endothelial growth factor (VEGF) overexpression [38].

\section{Conclusion}

Although an inexpensive, sensitive and specific serum test would be the most attractive approach to screen women for endometrial cancer, fundamental limitations 
of this method must be recognized. To achieve high specificity other modalities are likely to be required to be used in combination with serum markers, such as ultrasound and high-resolution MRI. Furthermore, patients at risk must be identified to improve the utility of such a test. For example, morbidly obese women have a risk of endometrial cancer that is ten times the risk of women of normal weight. Thus, a screen for endometrial cancer is likely to be most useful and most cost effective in obese women. Future efforts to diagnose endometrial cancer in early stages will be dependent not only on improving screening methods, but also on continued epidemiologic investigation. Recent breakthroughs in proteomics and bioinformatics technology will expand our understanding of tumor-specific biomarkers. Such investigations will establish newer and more useful biomarkers for the more accurate detection and management of endometrial cancers.

\section{REFERENCES}

[1] A. Jemal, R. Siegel, E. Ward, Y. Hao, J. Xu, T. Murray and M. J. Thun, “Cancer Statistics, 2008," CA: A Cancer Journal for Clinicians, Vol. 58, No. 2, 2008, pp. 71-96. doi:10.3322/CA.2007.0010

[2] V. E. von Gruenigen, K. M. Gil, H. E. Frasure, E. L. Jenison and M. P. Hopkins, "The Impact of Obesity and Age on Quality of Life in Gynecologic Surgery,” American Journal of Obstetrics \& Gynecology, Vol. 193, No. 4, 2005, pp. 1369-1375. doi:10.1016/j.ajog.2005.03.038

[3] F. Bray, A. H. Loos, M. Oostindier and E. Weiderpass, "Geographic and Temporal Variations in Cancer of the Corpus Uteri: Incidence and Mortality in Pre- and Postmenopausal Women in Europe," International Journal of Cancer, Vol. 20, No. 117, 2005, pp. 123-131. doi:10.1002/ijc.21099

[4] J. V. Bokhman, "Two Pathogenetic Types of Endometrial Carcinoma,” Gynecologic Oncology, Vol. 15, No. 1, 1983, pp. 10-17. doi:10.1016/0090-8258(83)90111-7

[5] B. A. Goff, D. Kato, R. A. Schmidt, M. Ek, J. A. Ferry, H. G. Muntz, J. M. Cain, H. K. Tamimi, D. C. Figge and B. E. Greer, "Uterine Papillary Serous Carcinoma: Patterns of Metastatic Spread,” Gynecologic Oncology, Vol. 54, No. 3, 1994, pp. 264-268. doi:10.1006/gyno.1994.1208

[6] K. Garg and R. A. Soslow, "Lynch Syndrome (Hereditary Non-Polyposis Colorectal Cancer) and Endometrial Carcinoma,” Journal of Clinical Pathology, Vol. 62, No 8, 2009, pp. 679-684. doi:10.1136/jcp.2009.064949

[7] E. Weiderpass, I. Persson, H. O. Adami, C. Magnusson, A. Lindgren and J. A. Baron, "Body Size in Different Periods of Life, Diabetes Mellitus, Hypertension, and Risk of Postmenopausal Endometrial Cancer (Sweden)," Cancer Causes \& Control, Vol.11, No. 2, 2000, pp. 185-192. doi:10.1023/A:1008946825313

[8] G. Kvale, I. Heuch and G. Ursin, "Reproductive Factors and Risk of Cancer of the Uterine Corpus: A Prospective Study," Cancer Research, Vol. 48, No. 21, 1988, pp. 6217-6221.
[9] P. Neven and I. Vergote, "Should Tamoxifen Users Be Screened for Endometrial Lesions?” Lancet, Vol. 17, No. 351, 1998, pp. 155-157. doi:10.1016/S0140-6736(05)78216-7

[10] Y. Ueda, T. Enomoto, T. Kimura, T. Miyatake, K. Yoshino, M. Fujita and T. Kimura, "Serum Biomarkers for Early Detection of Gynecologic Cancers,” Cancers, Vol. 2, No. 2, 2010, pp. 1312-1327. doi:10.3390/cancers2021312

[11] R. C. Bast Jr., S. Knauf, A. Epenetos, B. Dhokia, L. Daly, M. Tanner, J. Soper, W. Creasman, S. Gall, R. C. Knapp, et al., "Coordinate Elevation of Serum Markers in Ovarian Cancer But Not in Benign Disease,” Cancer, Vol. 15, No. 68, 1991, pp. 1758-1763. doi:10.1002/1097-0142(19911015)68:8<1758::AID-CNC R2820680819>3.0.CO;2-\#

[12] I. Jacobs and R. C. Bast Jr., "The CA 125 Tumour-Associated Antigen: A Review of the Literature," Human Reproduction, Vol. 4, No. 1, 1989, pp. 1-12.

[13] M. H. Vuento, U. H. Stenman, J. P. Pirhonen, J. I. Mäkinen, P. J. Laippala and T. A. Salmi, "Significance of a Single CA 125 Assay Combined with Ultrasound in the Early Detection of Ovarian and Endometrial Cancer," Gynecologic Oncology, Vol. 64, No. 1, 1997, pp. 141146. doi:10.1006/gyno.1996.4545

[14] G. Scambia, A. Gadducci, P. B. Panici, E. Foti, M. Ferdeghini, G. Ferrandina, M. Amoroso, C. Castellani, V. Facchini and S. Mancuso, "Combined Use of CA 125 and CA 15-3 in Patients with Endometrial Carcinoma," Gynecologic Oncology, Vol. 54, No. 3, 1994, pp. 292-297. doi:10.1006/gyno.1994.1213

[15] A. Hakala, B. M. Kacinski, E. R. Stanley, E. I. Kohorn, U. Puistola, J. Risteli, L. Risteli, C. Tomás and A. Kauppila, "Macrophage Colony-Stimulating Factor 1, a Clinically Useful Tumor Marker in Endometrial Adenocarcinoma: Comparison with CA 125 and the Aminoterminal Propeptide of Type III Procollagen,” American Journal of Obstetrics \& Gynecology, Vol. 173, No. 1, 1995, pp. 112119. doi:10.1016/0002-9378(95)90178-7

[16] P. L. Cherchi, S. Dessole, G. A. Ruiu, G. Ambrosini, M. Farina, G. Capobianco and A. Ambrosini, "The Value of Serum CA 125 and Association CA 125/CA 19-9 in Endometrial Carcinoma,” European Journal of Gynaecological Oncology, Vol. 20, No. 4, 1999, pp. 315-317.

[17] S. Ginath, J. Menczer, Y. Fintsi, E. Ben-Shem, M. Glezerman and I. Avinoach, "Tissue and Serum CA 125 Expression in Endometrial Cancer," International Journal of Gynecological Cancer, Vol. 12, No. 4, 2002, pp. 372375. doi:10.1046/j.1525-1438.2002.01007.X

[18] H. H. Chung, J. W. Kim, N. H. Park, Y. S. Song, S. B. Kang and H. P. Lee, "Use of Preoperative Serum CA-125 Levels for Prediction of Lymph Node Metastasis and Prognosis in Endometrial Cancer," Acta Obstetricia et Gynecologica Scandinavica, Vol. 85, No. 12, 2006, pp. 1501-1505. doi:10.1080/00016340601022777

[19] E. P. Beck, M. Wagner, L. Anselmino, F. Xu, R. C. Bast Jr. and W. Jaeger, "Is OVX1 a Suitable Marker for Endometrial Cancer?” Gynecologic Oncology, Vol. 65, No. 2, 1997, pp. 291-296. doi:10.1006/gyno.1997.4620 
[20] T. Kurihara, H. Mizunuma, M. Obara, K. Andoh, Y. Ibuki and T. Nishimura, "Determination of a Normal Level of Serum CA 125 in Postmenopausal Women as a Tool for Preoperative Evaluation and Postoperative Surveillance of Endometrial Carcinoma," Gynecologic Oncology, Vol. 69, No. 3, 1998, pp. 192-196. doi:10.1006/gyno.1998.5018

[21] M. Takami, H. Sakamoto, K. Ohtani, T. Takami and K. Satoh, “An Evaluation of CA125 Levels in 291 Normal Postmenopausal and 20 Endometrial AdenocarcinomaBearing Women before and after Surgery," Cancer Letters, Vol. 16, No. 121, 1997, pp. 69-72. doi:10.1016/S0304-3835(97)00332-7

[22] A. K. Sood, R. E. Buller, R. A. Burger, J. D. Dawson, J. I. Sorosky and M. Berman, "Value of Preoperative CA 125 Level in the Management of Uterine Cancer and Prediction of Clinical Outcome,” Obstetrics \& Gynecology, Vol. 90, No. 3, 1997, pp. 441-447. doi:10.1016/S0029-7844(97)00286-X

[23] A. Yildiz, H. Yetimalar, B. Kasap, C. Aydin, S. Tatar, F. Soylu, F. S. Yildiz, "Preoperative Serum CA 125 Level in the Prediction of the Stage of Disease in Endometrial Carcinoma," The European Journal of Obstetrics \& Gynecology and Reproductive Biology, Vol. 164, No. 2, 2012, pp. 191-195. doi:10.1016/j.ejogrb.2012.05.038

[24] A. Gadducci, S. Cosio, A. Carpi, A. Nicolini and A. R. Genazzani, "Serum Tumor Markers in the Management of Ovarian, Endometrial and Cervical Cancer,” Biomedicine \& Pharmacotherapy, Vol. 58, No. 1, 2004, pp. 2438. doi:10.1016/j.biopha.2003.11.003

[25] P. M. Carpenter, G. P. Gamboa, G. E. Dorion, N. S. Ramsinghani, A. M. Aïssi and A. Manetta, "Radiation-Induced CA 125 Production by Mesothelial Cells," Gynecologic Oncology, Vol. 63, No. 3, 1996, pp. 328-332. doi:10.1006/gyno.1996.0331

[26] S. S. Lo, D. K. Cheng, T. Y. Ng, L. C. Wong, H. Y. Ngan, "Prognostic Significance of Tumour Markers in Endometrial Cancer," Tumor Biology, Vol. 18, No. 4, 1997, pp. 241-249. doi:10.1159/000218037

[27] M. S. Lundstrøm, C. K. Høgdall, A. L. Nielsen and H. C. Nyholm, "Serum Tetranectin and CA 125 in Endometrial Adenocarcinoma,” Anticancer Research, Vol. 20, No. 5, 2000, pp. 3903-3906.

[28] F. V. Price, S. K. Chambers, M. L. Carcangiu, E. I. Kohorn, P. E. Schwartz, J. T. Chambers, "CA 125 May Not Reflect Disease Status in Patients with Uterine Serous Carcinoma," Cancer, Vol. 82, No. 9, 1998, pp. 17201725. doi:10.1002/(SICI)1097-0142(19980501)82:9<1720::AID -CNCR19>3.0.CO;2-6

[29] D. Abramovich, M. Markman, A. Kennedy, K. Webster and J. Belinson, "Serum CA-125 as a Marker of Disease
Activity in Uterine Papillary Serous Carcinoma,” Journal of Cancer Research and Clinical Oncology, Vol. 125, No. 12, 1999, pp. 697-698. doi:10.1007/s004320050336

[30] M. Sawada, Y. Okudaira, Y. Matsui and Y. Shimizu, "Immunosuppressive Acidic Protein in Patients with Gynecologic Cancer,” Cancer, Vol. 15, No. 54, 1984, pp. 652-656. doi:10.1002/1097-0142(1984)54:4<652::AID-CNCR2820 540411>3.0.CO;2-C

[31] N. Takeshima, Y. Shimizu, S. Umezawa, Y. Hirai, J. T. Chen, I. Fujimoto, K. Yamauchi and K. Hasumi, "Combined Assay of Serum Levels of CA 125 and CA 19-9 in Endometrial Carcinoma,” Gynecologic Oncology, Vol. 54, No. 3, 1994, pp. 321-326. doi:10.1006/gyno.1994.1217

[32] H. Hareyama, N. Sakuragi, S. Makinoda and S. Fujimoto, "Serum and Tissue Measurements of CA 72-4 in Patients with Endometrial Carcinoma," Journal of Clinical Pathology, Vol. 49, No. 12, 1996, pp. 967-970. doi:10.1136/jcp.49.12.967

[33] A. Gadducci, M. Ferdeghini, C. Prontera, P. Giordano, R. Cristofani, R. Bianchi and P. Fioretti, “A Comparison of Pretreatment Serum Levels of Four Tumor Markers in Patients with Endometrial and Cervical Carcinoma," European Journal of Gynaecological Oncology, Vol. 11, No. 4, 1990, pp. 283-288.

[34] C. S. Diefenbach, Z. Shah, A. Iasonos, R. R. Barakat, D. A. Levine, C. Aghajanian, P. Sabbatini, M. L. Hensley, J. Konner, W. Tew, D. Spriggs, M. Fleisher, H. Thaler and J. Dupont, "Preoperative Serum YKL-40 Is a Marker for Detection and Prognosis of Endometrial Cancer," Gynecologic Oncology, Vol. 104, No. 2, 2007, pp. 435-442. doi:10.1016/j.ygyno.2006.08.028

[35] R. G. Moore, A. K. Brown, M. C. Miller, D. Badgwell, Z. Lu, W. J. Allard, C. O. Granai, R. C. Bast Jr. and K. Lu, "Utility of a Novel Serum Tumor Biomarker HE4 in Patients with Endometrioid Adenocarcinoma of the Uterus," Gynecologic Oncology, Vol. 110, No. 2, 2008, pp. 196201. doi:10.1016/j.ygyno.2008.04.002

[36] M. Suzuki, M. Ohwada, I. Sato and M. Nagatomo, "Serum Level of Macrophage Colony-Stimulating Factor as a Marker for Gynecologic Malignancies,” Oncology, Vol. 52, No. 2, 1995, pp. 128-133. doi:10.1159/000227443

[37] R. Konno, T. Takano, S. Sato and A. Yajima, "Serum Soluble Fas Level as a Prognostic Factor in Patients with Gynecological Malignancies,” Clinical Cancer Research, Vol. 6, No. 9, 2000, pp. 3576-3580.

[38] B. Dobrzycka, S. J. Terlikowski, M. Kwiatkowski, M. Garbowicz, M. Kinalski and L. Chyczewski "Prognostic Significance of VEGF and Its Receptors in Endometrioid Endometrial Cancer," Ginekologia Polska, Vol. 81, No. 6, 2010, pp. 422-425. 